\title{
Is there a relationship between admission blood glucose level following acute poisoning and clinical outcome?
}

\author{
Ali Mohammad Sabzghabaee ${ }^{1}$, Nastaran Eizadi-Mood², Farzad Gheshlaghi², Nooshin Adib³ \\ Leila Safaeian ${ }^{4}$
}

\author{
${ }^{1}$ Isfahan Clinical Toxicology Research Center, Isfahan University of Medical Sciences, \\ Isfahan, Iran \\ 2Department of Clinical Toxicology, Noor and Ali Asghar (PBUH) Medical Center, Isfahan \\ University of Medical Sciences, Isfahan, Iran \\ ${ }^{3}$ Medical Intern, Isfahan University of Medical Sciences, Isfahan, Iran \\ ${ }^{4}$ Department of Food and Drug Administration, Isfahan University of Medical Sciences, \\ Isfahan, Iran
}

Submitted: 11 November 2009

Accepted: 11 February 2010

Arch Med Sci 2011; 7, 1: 81-86

DOI: 10.5114/aoms.2011.20608

Copyright @ 2011 Termedia \& Banach

\begin{abstract}
Introduction: The aim of this study was to investigate the relationship between the admission blood glucose level following acute poisoning, severity of acute poisoning and clinical outcome.

Material and methods: This prospective study was conducted on 345 deliberate self-poisoning patients. Standard demographic and clinical information; admission blood glucose level; poisoning severity score and outcome were recorded. Patients with a history of diabetes mellitus, receipt of pre-sampling intravenous dextrose solution or glucocorticoids, and poisoning with toxic agents which produce hyper- or hypoglycaemia were excluded.

Results: Mean age of the patients was $27.5 \pm 8.6$ years. Females outnumbered males (57.9\%). Oral ingestion of more than one drug (46.7\%) and opiates (14.2\%) were the main causes of poisoning. Blood glucose values ranged from $50 \mathrm{mg} / \mathrm{dl}$ to $396 \mathrm{mg} / \mathrm{dl}$. Hyper- and hypoglycaemia were observed in $23.8 \%$ and $13.91 \%$ respectively. A total of $24.41 \%$ and $22.92 \%$ of the patients in hyper- and hypoglycaemic groups had grade 3 and 4 severity score in comparison with $4.18 \%$ in the normoglycaemic group. Development of complications and death were $14.64 \%$ and $10.42 \%$ in patients with hyper- and hypoglycaemia versus $3.73 \%$ in patients with normoglycaemia. A significant difference between normoglycaemic and hyperglycaemic patients in the severity of poisoning and clinical outcome was observed $(P<0.001)$.

Conclusions: Admission blood glucose levels may have a relationship with the severity of poisoning and clinical outcome following acute poisoning.
\end{abstract}

Key words: blood glucose, acute poisoning, outcome, poisoning severity score, hyperglycaemia.

\section{Introduction}

Hyperglycaemia is a common problem in hospitalized patients with or without a history of diabetes mellitus [1, 2]. The increased requirement of the brain, heart, immune system and other tissues for glucose during stressful situations is associated with alteration in metabolic activity [3]. It has been proposed that stress has major effects on glucose metabolism through the activation and interaction of immunoneuroendocrine systems which result

\author{
Corresponding author: \\ Nastaran Eizadi-Mood MD, PhD \\ Department of Clinical \\ Toxicology, Noor and Ali \\ Asghar (PBUH) Medical Center \\ Isfahan University of Medical \\ Sciences \\ Ostandari St. \\ Isfahan, Iran \\ Phone: +98 3112241130 \\ E-mail: izadi@med.mui.ac.ir
}


in hyperglycaemia and insulin resistance [4]. The major causes of hyperglycaemia include increased release of stress hormones referring to counterregulatory hormones (epinephrine, norepinephrine, glucagon, growth hormone, beta-endorphin and cortisol), overproduction of proinflammatory cytokines (especially tumour necrosis factor- $\alpha$, interleukin-1 and -6), iatrogenic factors (administration of steroids, catecholamines and excess dextrose by total parental nutrition) and underlying disease states [5-10]. A growing body of evidence suggests that hyperglycaemia is associated with increased morbidity and mortality, and poor prognostic outcomes in critical illness [11-13].

It is noteworthy that various therapeutic agents have been implicated as diabetogenic factors. These drugs influence glucose metabolism and cause hyperglycaemia through multiple mechanisms of action [14]. Although marked blood glucose levels are generally uncommon with therapeutic doses of most of these medications, higher doses and intoxications are more likely to be accompanied by severe hyperglycaemia. The most common medications are shown in Table I [15-18]. Moreover, one of the reported adverse effects in organophosphate, carbamate and amitraz poisoning is hyperglycaemia [19-21].

On the other hand, several medications may reduce blood glucose levels. These agents are also shown in Table I [14].

Despite extensive clinical investigations about hyperglycaemia in both medical and surgical intensive care units (ICUs), to our best knowledge, stress hyperglycaemia due to poisoning has not

Table I. Drugs which influence blood glucose level and were excluded from this study

\begin{tabular}{|lc|}
\hline Hyperglycaemic drugs & Hypoglycaemic drugs \\
\hline Calcium channel blockers & Salicylates \\
\hline Glucocorticoids & $\beta$-2 agonists \\
\hline Hydrochlorothiazide & Acetaminophen \\
\hline Isoniazid & Non-selective $\beta$-blockers \\
\hline Chlorpromazine & Quinine \\
\hline$\beta$-adrenergic agonists & Quinidine \\
\hline$\beta$-blockers & Captopril \\
\hline Niacin & Imipramine \\
\hline Theophylline & Clofibrate \\
\hline Morphine & \\
\hline Amoxapine & \\
\hline Dopamine and its analogues & \\
\hline Organophosphate & \\
\hline Carbamate & \\
\hline Amitraz & \\
\hline
\end{tabular}

been described previously. Also we have not found any study on poisoned patients to show the admission blood glucose level with respect to poisoning severity score and outcome especially when medication-induced hyperglycaemia or hypoglycaemia were excluded.

Therefore the purpose of this study was to describe the relationship between admission blood glucose levels with severity of poisoning and clinical outcome following acute poisoning.

\section{Material and methods}

This prospective study was conducted in the Department of Poisoning Emergency and Clinical Toxicology of Noor Hospital, Isfahan University of Medical Sciences (Iran) from March 2005 to May 2005. A total of 345 adult deliberate self-poisoning patients, aged 18 years or over, were included. Exclusion criteria were a history of diabetes mellitus, receipt of presampling intravenous dextrose solution or glucocorticoids, and poisoning with medications or toxic agents which produce hyperor hypoglycaemia as mentioned in the introduction. The protocol was approved by the bioethics committee of the Isfahan University of Medical Sciences.

\section{Data collection}

Demographic and clinical information, including toxic agent, the time elapsed from poisoning until arrival at the emergency department, severity of the poisoning and outcomes were recorded prospectively in a dedicated database.

The clinical severity of poisoning was graded according to the method described by Persson and coworkers, and developed by the International Program on Chemical Safety, the European Community, and the European Association of Poisons Centres and Clinical Toxicologists (IPCS/EC/EAPCCT). The poisoning severity score (PSS) is a four-scale grading as (0) none, (1) minor, (2) moderate, (3) severe, and (4) fatal [22, 23]. It was determined at the time of initial inquiry and following recovery, using examination findings including the Glasgow coma score, pupil size, convulsion, respiratory rate, pulse rate, blood pressure, body temperature, electrocardiogram (ECG) findings, pain assessment, and laboratory presentations (serum glucose, potassium, sodium, $\mathrm{pH}$, and bicarbonate in arterial blood gases).

All patients received medical treatment under the direction of the hospitals' consultant physicians. The clinical outcome measures were described as recovery without complications, complications (respiratory, renal and liver failure) and death. 
Respiratory failure was diagnosed by monitoring arterial blood gases (ABGs) and chest X-ray. Also patients who were hypoxic in spite of oxygen through nasal cannula received oxygen by mask (5-10 l/min). The indication for intubation was hypoxia unresponsive to supplemental oxygen through nasal cannula or mask. Patients who failed to respond to supplemental oxygen were intubated and ventilated under the direction of critical care physicians. Acute renal failure was defined as serum creatinine $>2.0 \mathrm{mg} / \mathrm{dl}$. Liver failure was defined as highly elevated serum aminotransferase enzymes (compared to the normal baseline).

\section{Glucose measurements}

Blood samples were obtained on admission and if there was hyperglycaemia, subsequent blood samples were taken every $4 \mathrm{hr}$ for $12 \mathrm{hr}$ or more if needed. In cases of hypoglycaemia, glucose level was checked every hour by glucometer until the normal blood glucose levels were obtained. The serum glucose level was measured using the glucose oxidase method on a Technicon RA-1000 Auto Analyzer (Bayer Diagnostics, Ireland). The range of admission blood glucose levels was divided into hypoglycaemic (< $70 \mathrm{mg} / \mathrm{dl}$ ), normoglycaemic (70-126 mg/dl), and hyperglycaemic (> $126 \mathrm{mg} / \mathrm{dl}$ ) ranges [24].

\section{Statistical analysis}

Glucose values were evaluated as a continuous variable and also as hypoglycaemic, normoglycaemic and hyperglycaemic grades. Statistical analysis was performed by $\chi^{2}$, Mann-Whitney $U$ and Kruskal Wallis, and Spearman correlation tests using the SPSS 13.0 software. $P<0.05$ was considered as the statistically significant level.

\section{Results}

\section{Baseline characteristics}

Demographic and clinical characteristics of 345 patients who met the inclusion criteria for the study are presented in Table II. Females outnumbered males and mean age was $27.5 \pm 8.6$ years (range: $18-65$ years). Multi-drug abuse was the main cause of poisoning in the studied population (46.7\%). Opiates (14.2\%) and benzodiazepines (11.9\%) were the most frequently used drugs. The majority of cases $(50 \%)$ were grade 0 PSS (no symptoms or signs) and only $11 \%$ and $0.6 \%$ were grade 3 and 4 , respectively (severe and fatal).

\section{Clinical outcomes}

Complete recovery was observed in $92.7 \%$ of patients, $6.7 \%$ developed complications and $0.6 \%$ died. Aspiration pneumonitis was the most
Table II. Baseline and clinical characteristics of patients

\begin{tabular}{|c|c|}
\hline No. of patients & 345 \\
\hline Age [year] & $27.5 \pm 8.6$ \\
\hline \multicolumn{2}{|l|}{ Gender } \\
\hline Female & $200(57.9)$ \\
\hline Time elapsed after poisoning [hour] & $2.8 \pm 0.34$ \\
\hline \multicolumn{2}{|l|}{ Clinical status } \\
\hline Aware & $164(47.5)$ \\
\hline Sleepy & $67(19.4)$ \\
\hline Stupor & $82(23.8)$ \\
\hline Coma & $32(9.3)$ \\
\hline Admission blood glucose [mg/dl] & $115.9 \pm 3.9$ \\
\hline \multicolumn{2}{|l|}{ Severity of poisoning } \\
\hline No symptoms or signs & $169(50)$ \\
\hline Mild & $78(22.6)$ \\
\hline Moderate & $58(16.8)$ \\
\hline Severe & $38(11)$ \\
\hline Fatal & $2(0.6)$ \\
\hline \multicolumn{2}{|l|}{ Outcome } \\
\hline Death & $2(0.6)$ \\
\hline Recovery & $320(92.7)$ \\
\hline Complications & $23(6.7)$ \\
\hline Respiratory failure & $13(3.8)$ \\
\hline Renal failure & $6(1.7)$ \\
\hline Liver failure & $4(1.2)$ \\
\hline \multicolumn{2}{|l|}{ Substance (toxins) } \\
\hline Multi-drug & $161(46.7)$ \\
\hline Opiates & $49(14.2)$ \\
\hline Benzodiazepines & $41(11.9)$ \\
\hline Analgesics & $23(6.7)$ \\
\hline Antiepileptics & $19(5.5)$ \\
\hline Antidepressants & $9(2.6)$ \\
\hline Psychoactives & $8(2.3)$ \\
\hline Cardiovascular & $8(2.3)$ \\
\hline Cannabinoids & $7(2.0)$ \\
\hline Corrosives & $7(2.0)$ \\
\hline Alcohols & $6(1.7)$ \\
\hline Other & $7(2.0)$ \\
\hline
\end{tabular}

Data are expressed as mean \pm SEM or $n(\%)$. Multi-drug poisoning included various combinations of sedative-hypnotic, antidepressant, antipsychotic, analgesic, and antiepileptic drugs

frequently observed complication. The PSS was significantly correlated with clinical outcomes (Spearman correlation coefficient $=0.371$, $p<0.001)$. 


\section{Blood glucose levels}

Blood glucose values ranged from $50 \mathrm{mg} / \mathrm{dl}$ to 396 mg/dl (mean $115.9 \pm 3.9$ mg/dl). Hyperglycaemia and hypoglycaemia were observed in $23.8 \%$ and $13.91 \%$ respectively. In all hyperglycaemic patients, subsequent measurements of blood glucose show a trend to attenuation and normoglycaemia after supportive care. No medications were used to control hyperglycaemia. All patients with hypoglycaemia received infusion of $0.5-1 \mathrm{~g} / \mathrm{kg}$ of hypertonic dextrose to obtain a normal blood glucose level. The incidence of hypo-, normo- and hyperglycaemic cases in various grades of poisoning and clinical outcomes are shown in Tables III and IV. The correlations of blood glucose levels with other collected data were evaluated. There was no significant correlation using Spearman correlation test between blood glucose level and age $(p=0.13)$, gender $(p=0.054)$, time of arrival $(p=0.092)$ and toxin agent $(p=0.32)$. A total of $24.41 \%$ and $22.92 \%$ of the patients in hyper- and hypoglycaemic groups had grade 3 and 4 severity score in comparison with $4.18 \%$ in the normoglycaemic group (Table III). Development of complications and death were $14.64 \%$ and $10.42 \%$ in patients with hyper- and hypoglycaemia versus $3.73 \%$ in patients with normoglycaemia. A significant difference between normoglycaemic and hyperglycaemic patients was observed in the severity of poisoning and clinical outcome $(P<0.001)$ (Table IV, Figure 1$)$.

\section{Discussion}

In this prospective study, significant differences in the severity of poisoning were observed in the hyperglycaemic patients compared with normo- glycaemic patients. The patients with hypo- and hyperglycaemia showed higher grades of PSS. Cases in grade 4 (fatal) were only in the hyperglycaemic group. Adverse clinical outcome (complications and death) was more often present in patients with hyperglycaemia. Evaluation of acute poisoning induced hyperglycaemia revealed that admission blood glucose levels may be a predictor of hospital morbidity and mortality following acute poisoning.

Demographic and clinical data of the present study showed that young people and females were more vulnerable to self-poisoning. In other studies, suicide has also been mostly dominant in young adults. Gender differences have been reported in some studies and most of the self-poisoned patients have been female $[25,26]$. Multi-drug poisoning was found to be the most common type of poisoning. Because most of the patients were admitted within early hours after intoxication, there was no significant correlation between time of poisoning and hyperglycaemia.

Since all medications poisoning by which is known to influence blood glucose levels were excluded from our study, there was no significant correlation between toxin agent and admission blood glucose level. Because of exclusion of diabetic patients and poisoning with hyperglycaemic agents, it may be concluded that hyperglycaemia was induced by stress of poisoning. Attenuation of blood glucose levels in subsequent measurements in hyperglycaemic patients after supportive care also suggests the role of stress in the hyperglycaemic condition in some poisoned patients. Most patients improved completely without complications, and the mortality rate was $0.6 \%$. It is noteworthy that several high-risk patients, who had made a suicide

Table III. Admission blood glucose levels in the poisoning severity score groups

\begin{tabular}{|c|c|c|c|c|c|c|}
\hline \multirow{2}{*}{$\begin{array}{l}\text { Blood glucose } \\
\text { [mg/dl] }\end{array}$} & \multicolumn{5}{|c|}{ Poisoning severity scores } & \multirow[t]{2}{*}{ Total } \\
\hline & 0 & 1 & 2 & 3 & 4 & \\
\hline $70-126$ & $130(60.47)$ & $50(23.25)$ & $26(12.10)$ & $9(4.18)$ & & $215(100)$ \\
\hline$<70$ & $7(14.58)$ & $14(29.16)$ & $16(33.34)$ & $11(22.92)$ & & $48(100)$ \\
\hline$>126$ & $32(39.01)$ & $14(17.07)$ & $16(19.51)$ & $18(21.95)$ & $2(2.46)$ & $82(100)$ \\
\hline
\end{tabular}

Poisoning severity score was graded as (0) none, (1) minor, (2) moderate, (3) severe, and (4) fatal. Data are expressed as $n$ (\%)

Table IV. Poisoning severity score was graded as (0) none, (1) minor, (2) moderate, (3) severe, and (4) fatal

\begin{tabular}{|lccc|}
\hline $\begin{array}{l}\text { Blood glucose } \\
{[\mathrm{mg} / \mathrm{dl}]}\end{array}$ & 1 & Clinical outcome & 3 \\
\cline { 2 - 4 } & $207(96.27)$ & 2 & Total \\
\hline $70-126$ & $43(89.58)$ & $8(3.73)$ & $215(100)$ \\
\hline$<70$ & $70(85.36)$ & $5(10.42)$ & $2(2.44)$ \\
\hline$>126$ & $10(12.20)$ & $82(100)$ \\
\hline
\end{tabular}

Clinical outcome was graded as (1) recovery without complications, (2) recovery with complications, and (3) death. Data are expressed as $n$ (\%) 
attempt with an antihypertensive medication or some insecticides and pesticides (as a predictor of ICU admission) were excluded from this study because of induction of glucose tolerance disorders $[25,27]$.

Although poisoning is one of the important causes of significant morbidity and mortality, and appropriate management is very important in critically ill poison patients [28], acute poisoninginduced hyperglycaemia has not been previously studied in these patients. The results of various studies in critically ill patients have shown that stress-induced hyperglycaemia is a strong predictor of increased mortality and adverse clinical outcome $[1,5,13]$. Hyperglycaemia is found to be associated with increased risk of infectious complications and septic shock, reduced immune response, dehydration and electrolyte imbalances and lethal multiple organ failure in traumatic and acute ischaemic events [12, 29, 30].

Some toxicological studies have been performed on medications or toxic agents which influence blood glucose levels. Hyperglycaemia has been related to adverse neurological outcome and brain damage in acute severe carbon monoxide poisoning [31]. In acute aluminium phosphide poisoning, hyperglycaemia as a potential prognostic factor has been correlated with mortality [32]. Hyperglycaemia is also the outcome of acute or chronic exposure to organophosphorus compounds [19]. In overdoses of calcium channel blocker agents, serum glucose concentrations correlate directly with the severity of the calcium channel blocker intoxication [33].

Various clinical investigations in both medical and surgical ICUs have confirmed that maintaining normoglycaemia can improve the prognosis in critical illness $[1,5,11]$. Insulin remains the obvious treatment for hyperglycaemia and lowering blood glucose levels to $80-110 \mathrm{mg} / \mathrm{dl}$ has a pivotal role in the treatment of such patients [34]. Prevention of the inflammatory process and insulin resistance may also be important for the improvement of critically ill patients with acute hyperglycaemia $[5,35]$. Although the role of treatment of hyperglycaemia with insulin and metformin has been evaluated in critically ill patients in the studies by Goldberg and Mojtahedzadeh, none of these modalities was performed in poisoning cases. A large randomized controlled trial study is necessary to affirm the role of insulin or metformin treatment in admission hyperglycaemia induced by severe poisoning.

Although poisoning cases with toxic agents which produce hypoglycaemia were excluded from our study, hypoglycaemia was also observed in $13.91 \%$ of patients. However, there was no relationship between hypoglycaemia and severity of poisoning. We have no explanation for this. Since

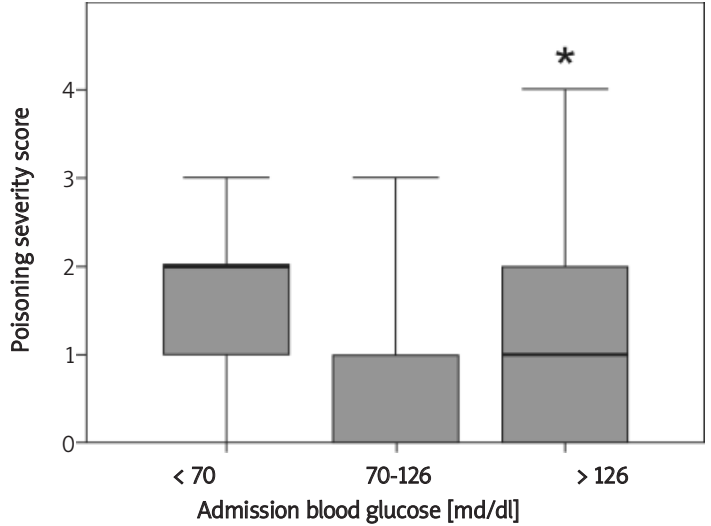

Figure 1. Correlation between admission blood glucose levels with poisoning severity scores Poisoning severity score was graded as (0) none, (1) minor, (2) moderate, (3) severe, and (4) fatal. P values are from Kruskal-Wallis non-parametric tests. Thick lines represent the median grade of each group, boxes show the interquartile range and bars represent the maximum and minimum sample values.

${ }^{*} p<0.001$ comparing hyperglycaemic patients (> 126) versus normoglycaemic patients $(70-126 \mathrm{mg} / \mathrm{dl})$

hypoglycaemia recovered after $0.5-1 \mathrm{~g} / \mathrm{kg}$ infusion of hypertonic dextrose, the poor nutrition of suicidal patients may be one of the reasons (because of an argument with their family they had not eaten lunch or dinner before the suicide attempt).

In conclusion, acute poisoning-induced hyperglycaemia may be a good predictor of severity of poisoning and clinical outcome. However, further studies for the optimal care of critically poisoned patients with hyperglycaemia and multi-disciplinary approaches to decrease the mortality rate among these patients are needed.

The main limitation of our study is that it was performed in a single medical centre in a nonrandomized design and underlying disease states as a risk factor for hyperglycaemia were not considered other than diabetes mellitus.

\section{Acknowledgments}

This research was result of an approved medical internship thesis and was designed, and financially supported by the Isfahan clinical toxicology research center (http://ctrc.mui.ac.ir). The authors would like to thank the academic faculty members of the anaesthesiology department at Isfahan University of Medical Sciences and all of the colleagues and staff in the Clinical Toxicology Department of Noor and Ali Asghar (PBUH) Hospital for their valuable endeavours and support.

\section{References}

1. Lonergan T, Compte AL, Willacy M, et al. A pilot study of the SPRINT protocol for tight glycemic control in critically ill patients. Diabetes Technol Ther 2006; 8: 449-62. 
2. Boord JB, Greevy RA, Braithwaite SS, et al. Evaluation of hospital glycemic control at US Academic Medical Centers. J Hosp Med 2009; 4: 35-44.

3. Rashid S, Shi ZQ, Niwa M, et al. Beta-blockade, but not normoglycemia or hyperinsulinemia, markedly diminishes stress-induced hyperglycemia in diabetic dogs. Diabetes 2000; 49: 253-62.

4. Beishuizen A, Thijs LG. The immunoneuroendocrine axis in critical illness: beneficial adaptation or neuroendocrine exhaustion? Curr Opin Crit Care 2004; 10: 461-7.

5. Kajbaf F, Mojtahedzadeh M, Abdollahi M. Mechanisms underlying stress-induced hyperglycemia in critically ill patients. Therapy 2007; 4: 97-106.

6. Mizock BA. Alterations in fuel metabolism in critical illness: hyperglycaemia. Best Pract Res Clin Endocrinol Metab 2001; 15: 533-51.

7. McCowen KC, Malhotra A, Bistrian BR. Stress-induced hyperglycemia. Crit Care Clin 2001; 17: 107-24.

8. Imura $\mathrm{H}$, Fukata J. Endocrine-paracrine interaction in communication between the immune and endocrine systems. Activation of the hypothalamic-pituitary-adrenal axis in inflammation. Eur J Endocrinol 1994; 130: 32-7.

9. Chrousos GP. The hypothalamic-pituitary-adrenal axis and immune-mediated inflammation. N Engl J Med 1995; 332: 1351-62.

10. Rosmarin DK, Wardlaw GM, Mirtallo J. Hyperglycemia associated with high, continuous infusion rates of total parenteral nutrition dextrose. Nutr Clin Pract 1996; 11: 151-6.

11. Gearhart MM, Parbhoo SK. Hyperglycemia in the critically ill patient. AACN Clin Issues 2006; 17: 50-5.

12. Weekers F, Giulietti AP, Michalaki M, et al. Metabolic, endocrine, and immune effects of stress hyperglycemia in a rabbit model of prolonged critical illness. Endocrinology 2003; 144: 5329-38.

13. Capes SE, Hunt D, Malmberg K, Pathak P, Gerstein HC. Stress hyperglycemia and prognosis of stroke in nondiabetic and diabetic patients: a systematic overview. Stroke 2001; 32: 2426-32.

14. Pandit MK, Burke J, Gustafson AB, Minocha A, Peiris AN. Drug-induced disorders of glucose tolerance. Ann Intern Med 1993; 118: 529-39.

15. Erle G, Basso M, Federspil G, Sicolo N, Scandellari C. Effect of chlorpromazine on blood glucose and plasma insulin in man. Eur J Clin Pharmacol 1977; 11: 15-8.

16. Kline JA, Raymond RM, Schroeder JD, Watts JA. The diabetogenic effects of acute verapamil poisoning. Toxicol Appl Pharmacol 1997; 145: 357-62.

17. Malyuk R, Gibson B, Procyshyn RM, Kang N. Olanzapine associated weight gain, hyperglycemia and neuroleptic malignant syndrome: case report. Int J Geriatr Psychiatry 2002; 17: 326-8.

18. Polak M, Rolon MA, Chouchana A, Czernichow P. Theophylline intoxication mimicking diabetic ketoacidosis in a child. Diabetes Metab 1999; 25: 513-5.

19. Rahimi R, Abdollahi M. A review on the mechanisms involved in hyperglycemia induced by organophosphorus pesticides. Pesticide Biochemistry and Physiology 2007 88: $115-21$.

20. Weizman Z, Sofer S. Acute pancreatitis in children with anticholinesterase insecticide intoxication. Pediatrics 1992; 90: 204-6.

21. Demirel Y, Yilmaz A, Gursoy S, Kaygusuz K, Mimaroglu C. Acute amitraz intoxication: retrospective analysis of 45 cases. Hum Exp Toxicol 2006; 25: 613-7.

22. Persson HE, Sjoberg GK, Haines JA, Pronczuk de Garbino J. Poisoning severity score. Grading of acute poisoning. J Toxicol Clin Toxicol 1998; 36: 205-13.
23. Casey PB, Dexter EM, Michell J, Vale JA. The prospective value of the IPCS/EC/EAPCCT poisoning severity score in cases of poisoning. J Toxicol Clin Toxicol 1998; 36: 215-7.

24. Ousman Y. Hyperglycemia in the Hospitalized Patient. Clinical Diabetes 2002; 20: 147-8.

25. Novack V, Jotkowitz A, Delgado J, et al. General characteristics of hospitalized patients after deliberate self-poisoning and risk factors for intensive care admission. Eur J Intern Med 2006; 17: 485-9.

26. Paudyal BP. Poisoning: pattern and profile of admitted cases in a hospital in central Nepal. JNMA J Nepal Med Assoc 2005; 44: 92-6.

27. Sandhu JS, Dhiman A, Mahajan A, Sandhu P. Outcome of paraquat poisoning - a five year study. Indian journal of nephrology. 2003; 13: 64-8.

28. Chan $\mathrm{Y}$, Fung $\mathrm{H}$, Lee $\mathrm{C}$, et al. A prospective epidemiological study of acute poisoning in Hong Kong. Hong Kong Journal of Emergency Medicine 2005; 12: 156-61.

29. Ahmann A. Comprehensive management of the hospitalized patient with diabetes. The Endocrinologist 1998; 8: 250-9.

30. Malmberg K, Ryden L, Efendic S, et al. Randomized trial of insulin-glucose infusion followed by subcutaneous insulin treatment in diabetic patients with acute myocardial infarction (DIGAMI study): effects on mortality at 1 year. J Am Coll Cardiol 1995; 26: 57-65.

31. Penney DG. Hyperglycemia exacerbates brain damage in acute severe carbon monoxide poisoning. Med Hypotheses 1988; 27: 241-4.

32. Mehrpour O, Alfred S, Shadnia S, et al. Hyperglycemia in acute aluminum phosphide poisoning as a potential prognostic factor. Hum Exp Toxicol 2008; 27: 591-5.

33. Levine M, Boyer EW, Pozner CN, et al. Assessment of hyperglycemia after calcium channel blocker overdoses involving diltiazem or verapamil. Crit Care Med 2007; 35: 2071-5.

34. Goldberg PA, Siegel MD, Sherwin RS, et al. Implementation of a safe and effective insulin infusion protocol in a medical intensive care unit. Diabetes Care 2004; 27 : 461-7.

35. Mojtahedzadeh M, Rouini MR, Kajbaf F, et al. Advantage of adjunct metformin and insulin therapy in the management of glycemia in critically ill patients. Evidence for nonoccurrence of lactic acidosis and needing to parenteral metformin. Arch Med Sci 2008; 4: 174-81. 